\title{
O SISTEMA RADICULAR DA VARIEDADE RB855536 DE CANA-DE-AÇÚCAR NO PLANTIO EM MINITOLETE E TOLETE
}

\section{SUGARCANE ROOT SYSTEM OF RB855536 VARIETY PLANTED IN ONE BUD AND THREE BUDS SETTS}

\author{
Pedro Henrique de Medeiros BUSO ${ }^{1}$ \\ Henrique Soares KOEHLER ${ }^{2}$ \\ Edelclaiton DAROS ${ }^{3}$ \\ José Luis Camargo ZAMBON ${ }^{3}$ \\ Oswaldo Teruyo IDO ${ }^{3}$ \\ João Carlos BESPALHOK-FILHO ${ }^{3}$ \\ Heroldo WEBER ${ }^{4}$ \\ Ricardo Augusto OLIVEIRA ${ }^{3}$ \\ Hugo ZENI NETO ${ }^{5}$
}

\section{RESUMO}

Este trabalho teve como objetivo estudar o desenvolvimento radicular de cana-de-açúcar em dois sistemas de plantio, minitolete e tolete. O experimento foi realizado na Estação Experimental de Paranavaí, no município de ParanavaíPR, Setor de Ciências Agrárias, Universidade Federal do Paraná, no período de 27 de junho de 2000 a 10 de julho de 2001. Foi utilizado delineamento em blocos ao acaso, com duas formas de plantio. Foram 9 épocas de avaliações durante o ciclo de 378 dias. A forma de plantio e a profundidade de amostragem não alteraram o comprimento e diâmetro radicular. Nos dois sistemas de plantio verificou-se que as variáveis superfície específica e volume radicular na camada $00-20 \mathrm{~cm}$ foram significativamente superiores. No sistema de plantio em minitolete, houve prioridade no crescimento radicular em profundidade, podendo ser uma característica importante para o desenvolvimento em condições de restrição hídrica.

Palavras-chave: sistema de plantio; raiz; Saccharum spp.

\begin{abstract}
This work aimed to study the sugarcane root development in two different planting systems: using one bud setts and three buds setts. The experiment took place in Estação Experimental de Paranavaí, county of Paranavaí-PR, Agricultural Science Sector, Federal University of Paraná during the period of June $27^{\text {th }} 2000$ until July $10^{\text {th }} 2001$. The delineation was design as random blocks, with two ways of planting. Evaluation was performed 9 times during the 378 days cycle. The way and the depth of planting did not alter the length and diameter of the root system. In the two ways of planting it was verified that the variables specific surface and root system volume in the $00-20 \mathrm{~cm}$ depth were significantly higher. In the one bud setts system, it was observed a priority of root growth in length, a characteristic that can be important to drought conditions.
\end{abstract}

Key-words: plant system; root; Saccharum spp.

\footnotetext{
${ }^{1}$ Engenheiro Agrônomo, M.Sc., Programa de Melhoramento Genético de Cana-de-açúcar, Departamento de Fitotecnia e Fitossanitarismo da Universidade Federal do Paraná (UFPR), Paranavaí, Paraná, Brasil, E-mail: pedrohbuso@hotmail.com.

2 Engenheiro Florestal, Doutor, Professor do Departamento de Fitotecnia e Fitossanitarismo da Universidade Federal do Paraná (UFPR), Curitiba, Paraná, Brasil, E-mail: koehler@ufpr.br.

${ }^{3}$ Engenheiro Agrônomo, Doutor, Professor do Departamento de Fitotecnia e Fitossanitarismo da Universidade Federal do Paraná (UFPR), Rua dos Funcionários, n. 1540, 80035-050, Cabral, Curitiba, Paraná, Brasil, E-mail: ededaros@ufpr.br; joseluis@ufpr.br; ido@ufpr.br; bespa@ufpr.br; rico@ufpr.br. *Autor para correspondência: ededaros@ufpr.br.

${ }^{4}$ Engenheiro Agrônomo, Doutor, Universidade Federal do Paraná (UFPR), Paranavaí, Paraná, Brasil, E-mail: heroldo@ufpr.br.

${ }^{5}$ Engenheiro Agrônomo, M.Sc. Doutorando em Agronomia, Genética, Universidade Estadual de Maringá (UEM), Maringá, Paraná, Brasil, Email: neto_hugo@hotmail.com
} 
BUSO, P.H.M. et al. O sistema radicular da variedade...

\section{INTRODUÇÃO}

No Brasil, a cultura da cana-de-açúcar está instalada em uma área superior a 7 milhões ha, produzindo mais de 571 milhões de toneladas de cana, conforme dados da safra 2008 , destinados à fabricação de açúcar, álcool, aguardente e também para a alimentação de bovinos. No Estado do Paraná a cana-de-açúcar ocupa uma área próxima dos 509 mil ha, divididos entre 27 unidades correspondentes a usinas e destilarias (Conab, 2009; Alcopar, 2009).

A cana-de-açúcar é uma planta perene da família das Poaceaes e tem como características o perfilhamento abundante na fase inicial de crescimento, aliado ao intenso crescimento do sistema radicular fasciculado. Posteriormente, segue a fase de grande crescimento, com grande acúmulo de massa seca, tanto na parte aérea como no sistema radicular, seguido da fase final onde ocorre a maturação, ou seja, acúmulo de sacarose nos colmos. Entretanto, são vários os fatores que promovem a inibição ou favorecem o desenvolvimento da cultura (Oliveira et al., 2004; Ido et al., 2006).

Normalmente, as avaliações de variedades das diferentes culturas estão baseadas na parte aérea das plantas. Embora seja a extensão, a distribuição e a atividade das raízes que determinam a quantidade de água e de nutrientes absorvidos, essenciais para o desenvolvimento das culturas, pouco do sistema radicular tem sido estudado, negligenciando a sua importância (Vasconcelos \& Garcia, 2005).

Estudos do sistema radicular são de grande importância para a compreensão dos diversos fenômenos de desenvolvimento, mas exige procedimentos criteriosos. Além de trabalhosos, os parâmetros radiculares são influenciados pela variabilidade físico-química do solo, dentre outros fatores (Vasconcelos et al., 2003).

O objetivo deste trabalho foi avaliar o desenvolvimento radicular da variedade RB855536 em dois sistemas de plantio; minitoletes com apenas uma gema e convencional com plantio de toletes contendo três gemas.

\section{MATERIAL E MÉTODOS}

O experimento foi instalado na Estação Experimental de Paranavaí, pertencente ao Setor de Ciências Agrárias da UFPR, localizada na Região Noroeste do Estado do Paraná, no Município de Paranavaí, entre as coordenadas de $22^{0} 55^{\prime}$ de latitude Sul e $52^{0} 27^{\prime}$ de longitude Oeste, com altitude de $470 \mathrm{~m}$.

O solo é classificado como Latossolo Vermelho Distrófico, com relevo suave ondulado. Foi realizada calagem conforme análise de solo (Tabela 1), com adubação de base no sulco de plantio de $20 \mathrm{~kg} \mathrm{ha}^{-1}$ de N, $100 \mathrm{~kg} \mathrm{ha}^{-1}$ de $\mathrm{K}_{2} \mathrm{O}$ e 100 $\mathrm{kg} \mathrm{ha}^{-1}$ de $\mathrm{P}_{2} \mathrm{O}_{5}$. O plantio foi realizado no dia $06 \mathrm{de}$ abril de 1999.O espaçamento entrelinhas foi de 1,40 $\mathrm{m}$.

TABELA 1 - Resultados das análises química e granulométrica, Estação Experimental de Paranavaí, SCAUFPR, 1999.

\begin{tabular}{|c|c|c|c|c|c|c|c|c|c|c|c|}
\hline \multirow{3}{*}{ Camada } & \multicolumn{8}{|c|}{.Química.. } & \multicolumn{3}{|c|}{.....Granulométrica.. } \\
\hline & $\mathrm{pH}$ & $\mathrm{Al}^{3-}$ & $\mathrm{H}+\mathrm{Al}$ & $\mathrm{Ca}^{2+}$ & $\mathrm{Mg}^{2+}$ & $\mathrm{K}^{+}$ & \multirow{2}{*}{$\begin{array}{c}P \\
m g ~ d m^{-3}\end{array}$} & \multirow{2}{*}{$\begin{array}{c}C \\
g d^{-3}\end{array}$} & Areia & Silte & Argila \\
\hline & $\mathrm{CaCl}_{2}$ & $\ldots \ldots$ & .......cr & c. $\mathrm{dm}^{-}$ & & $\cdots$ & & & ......... & ( & \\
\hline $00-20 \mathrm{~cm}$ & 5,20 & 0,0 & 2,37 & 1,15 & 0,85 & 0,15 & 8,00 & 9,19 & 82 & 1 & 17 \\
\hline
\end{tabular}

O experimento foi conduzido sob delineamento experimental em blocos casualizados, em arranjo de parcelas subdivididas no tempo, com cinco repetições por época. Os tratamentos foram representados pelos dois sistemas de plantio; minitolete e tolete e em nove épocas de amostragens.

Foram realizadas coletas periódicas nas seguintes épocas: $00,54,92,126,161,210,252$, 316 e 378 dias após o corte (DAC) da cana-planta. Em cada época foram obtidas amostras das raízes, por meio da abertura de trincheiras localizadas próximas a cada touceira de cana-de-açúcar amostrada. As dimensões das trincheiras foram de $0,50 \mathrm{~m}$ de largura e 1,40 m de comprimento; $0,70 \mathrm{~m}$ para cada lado a partir do centro do sulco de plantio da cana. A profundidade da trincheira foi de $0,60 \mathrm{~m}$. As touceiras foram tomadas aleatoriamente na área útil da parcela experimental. No plantio em minitoletes foi retirada toda a touceira, e levadas para o laboratório. No plantio em toletes foi retirada toda cana encontrada a cada $0,50 \mathrm{~m}$ posterior à trincheira, perfazendo assim o mesmo volume amostrado no plantio em minitolete (um metro linear de plantio).

Para quantificar o sistema racidular nas nove épocas, coletou-se nas trincheiras e em três profundidades $(00-20,20-40$ e $40-60 \mathrm{~cm})$ amostras, utilizando cilindro volumétrico com $20 \mathrm{~cm}$ de altura, diâmetro interno de $4,5 \mathrm{~cm}$ e parede externa de 0,5 $\mathrm{cm}$, com borda serrilhada e cortante numa das extremidades (volume de $318 \mathrm{~cm}^{3}$ ).

As porções de solo e raiz das amostras foram enviadas para laboratório onde foram deixadas em $25 \mathrm{~cm}^{3}$ de hidróxido de sódio $1 \mathrm{~mol}$ $\mathrm{dm}^{-3}$ diluído em $1 \mathrm{dm}^{3}$ de água durante $12 \mathrm{~h}$ para a dispersão da argila, e em seguida lavagem em água corrente sobre jogo de peneiras com malhas de 0,50 e $0,25 \mathrm{~mm}$. A separação das raízes da canade-açúcar de outros materiais minerais e orgânicos 
BUSO, P.H.M. et al. O sistema radicular da variedade...

foi realizada manualmente com pinças de ponta fina em água. As raízes foram acondicionadas em potes plásticos contendo solução de etanol $50 \%$ e armazenados a $0{ }^{\circ} \mathrm{C}$ conforme Böhm (1979) até determinação dos seus parâmetros.

As amostras de raízes foram processadas por meio do programa computacional WinRhizo, acoplado a um Scaner LA1600 (Regent Instruments Inc., Canadá). Foram geradas as variáveis comprimento das raízes $(\mathrm{cm})$ e superfície específica $\left(\mathrm{cm}^{2}\right)$.

$\mathrm{Na}$ parte aérea das plantas foi realizada a contagem dos perfilhos do metro linear de sulco, estatura $(\mathrm{m})$ com auxílio de uma fita métrica, sendo considerado o colo da planta até o último colarinho visível (folha +1), número de folhas verdes totalmente abertas (desenvolvidas) por perfilho (da folha 0 até a folha +7 , do "Sistema Kuijper"), comprimento e largura $(\mathrm{cm})$ da folha +3 . Para estimar a área foliar adotou-se metodologia proposta por Hermann \& Câmara (1999), pela seguinte equação: $A F=C x L \times 0,75 x(N+2)$, onde $C$ é o comprimento da folha $+3, L$ é a largura da folha +3 , 0,75 é o fator de correção para área foliar da cultura e $N$ é o número de folhas abertas com pelo menos $20 \%$ de área verde.

As curvas de crescimento do sistema radicular, nas nove épocas, e da parte aérea da cana-soca em oito épocas, dos 54 DAC aos 378 DAC, foram ajustadas, em função do tempo, por equação exponencial quadrática, por meio do programa computacional Excel 2003.

Os resultados das avaliações de comprimento e superfície específica aos 378 DAC foram submetidos à análise de variância, utilizando o programa MSTAT-C ${ }^{\circledR}$. Quando estes dados foram significativos pelo teste $\mathrm{F}$, ao nível de $5 \%$ e $1 \%$ de probabilidade as médias foram comparadas pelo teste de Tukey, ao nível de probabilidade de 5\%.

\section{RESULTADOS E DISCUSSÃO}

A análise de variância revelou que os fatores formas de plantio e profundidade são independentes apenas para a variável comprimento de raízes. Ocorreram diferenças significativas somente para 0 fator profundidade na variável comprimento de raízes. Considerando esta característica, verificou-se que na profundidade 00 a $20 \mathrm{~cm}$ foram identificados valores estatisticamente superiores, quando comparado as demais profundidades (Tabela 2). Vasconcelos (1998), Ido et al. (2006) também relataram maior concentração radicular em comprimento nas camadas superiores do solo.

TABELA 2 - Comprimento $(\mathrm{cm})$ de raízes da variedade RB855536 em três profundidades aos 378 DAC. Estação Experimental de Paranavaí, SCA-UFPR, 2000/2001.

\begin{tabular}{|c|c|}
\hline PROFUNDIDADES (cm) & COMPRIMENTO $(\mathrm{cm})$ \\
\hline $00-20$ & $10,04 a^{*}$ \\
\hline $20-40$ & $3,46 \quad b$ \\
\hline $40-60$ & $2,47 \quad b$ \\
\hline $\mathrm{CV}$ & $37,58 \%$ \\
\hline DMS & 1,22 \\
\hline
\end{tabular}

* Médias seguidas da mesma letra na vertical não diferem estatisticamente pelo teste de Tukey a $5 \%$ de probabilidade.

Ao considerar os tratamentos principais para a característica superfície específica de raízes $\left(\mathrm{cm}^{2}\right)$, observou-se que no sistema de plantio em minitoletes (gema) houve desenvolvimento superior a $30 \%$ quando comparado com o sistema de plantio em toletes (convencional), na profundidade de 00 $20 \mathrm{~cm}$ (Tabela 3). Vasconcelos (1998), estudando o sistema radicular de quatro variedades de cana-deaçúcar em cana planta, nas profundidades 00-20 $\mathrm{cm}, 20-40 \mathrm{~cm}, 40-60 \mathrm{~cm}$ e 60-80 cm, também encontrou maior concentração de raízes em camadas mais superficiais, com $54,23 \%$ da massa de raízes concentradas na camada superficial do solo $(00-20 \mathrm{~cm})$.

TABELA 3 - Superfície específica $\left(\mathrm{cm}^{2}\right)$ de raízes da variedade RB855536 nos sistemas de plantio em minitolete e tolete, em três profundidades, 0-20, 20-40 e 40-60 (cm), aos 378 DAC. Estação Experimental de Paranavaí, SCA-UFPR, 2000/2001.

\begin{tabular}{|c|c|c|}
\hline TRATAMENTO & PROFUNDIDADES (cm) & SUPERFÍCIE ESPECÍFICA $\left(\mathrm{cm}^{2}\right)$ \\
\hline \multirow{3}{*}{ MINITOLETE } & $00-20$ & $349,86 a^{*}$ \\
\hline & $20-40$ & $60,22 \quad b$ \\
\hline & $40-60$ & 39,99 \\
\hline \multirow{3}{*}{ TOLETE } & $00-20$ & 260,70 a \\
\hline & $20-40$ & $144,32 \quad b$ \\
\hline & $40-60$ & 103,21 \\
\hline \multicolumn{2}{|c|}{$\mathrm{CV}$} & $31,43 \%$ \\
\hline \multicolumn{2}{|c|}{ DMS } & 65,23 \\
\hline
\end{tabular}

* Médias seguidas da mesma letra na vertical não diferem estatisticamente pelo teste de Tukey a $5 \%$ de probabilidade. 
BUSO, P.H.M. et al. O sistema radicular da variedade...

A variação no comprimento do sistema radicular, como observado na Figura 1, tende a ser normal segundo Aguiar (1978), que seria explicado devido a alternância de raízes vivas e mortas ao longo do ciclo. Aos 161 DAC houve comportamento descontínuo das raízes. Aparentemente, com a morte dos perfilhos (Figura 2) a seiva é translocada e redirecionada para os perfilhos sobreviventes, melhores dispostos e maiores (fonte/dreno), proporcionando rápido desenvolvimento destes. Neste caso, as raízes dos perfilhos fracos morrem e ocorre renovação de raízes, geradas pelos perfilhos restantes (Taylor \& Arkin, 1981), apesar de exibirem comportamento semelhantes os tratamentos tolete e minitolete entre as profundidades $20-40$ e $40-60$ (Figura 1).

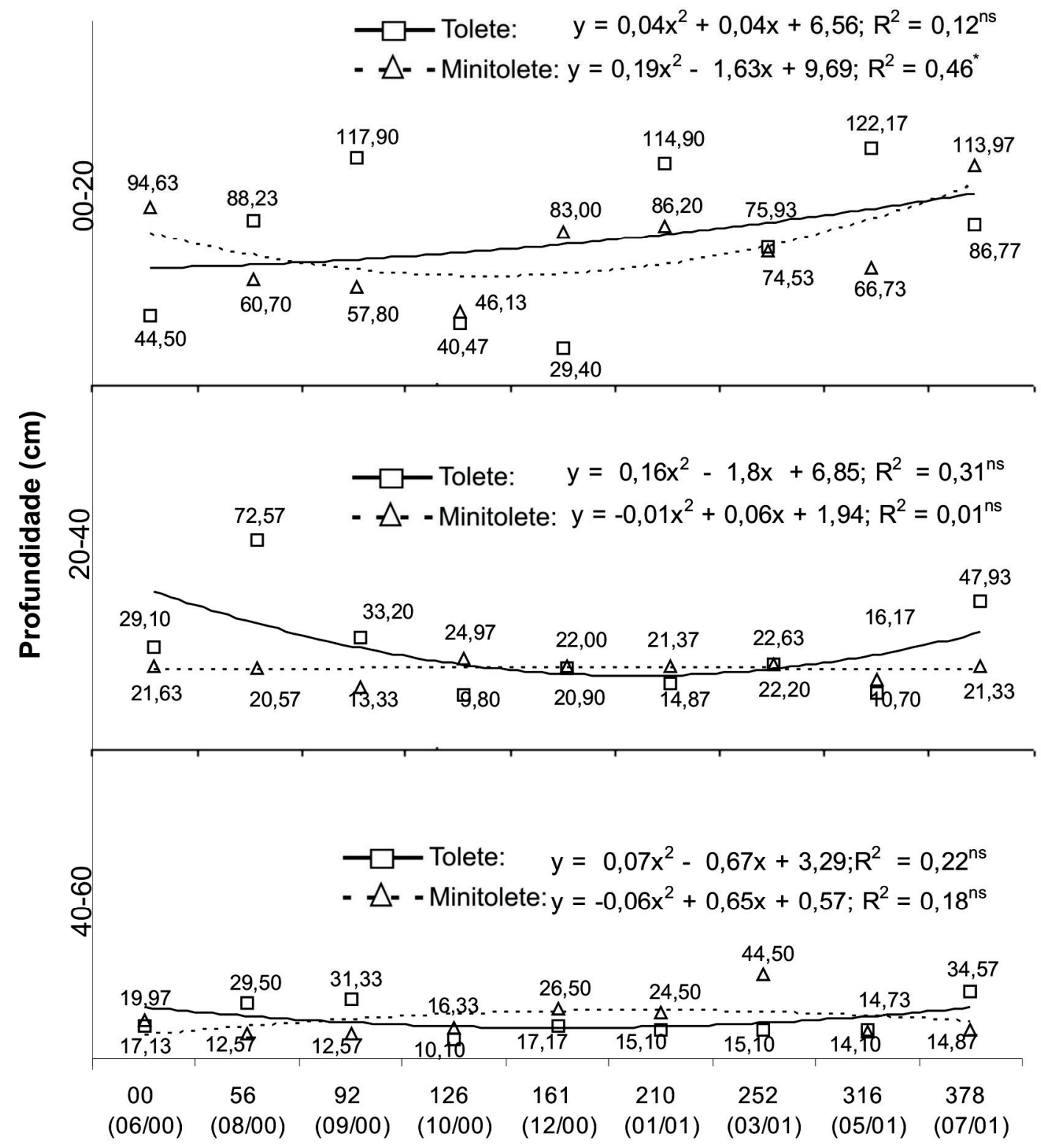

\section{Épocas em Dias}

FIGURA 1 - Comprimento de raízes (cm) nas profundidades 00-20, 20-40 e 40-60 (cm); em dois sistemas de plantio: tolete e minitolete, para a variedade RB855536, nas nove épocas de amostragens. Estação Experimental de Paranavaí, SCA-UFPR, PR, 2000/2001. 
BUSO, P.H.M. et al. O sistema radicular da variedade...

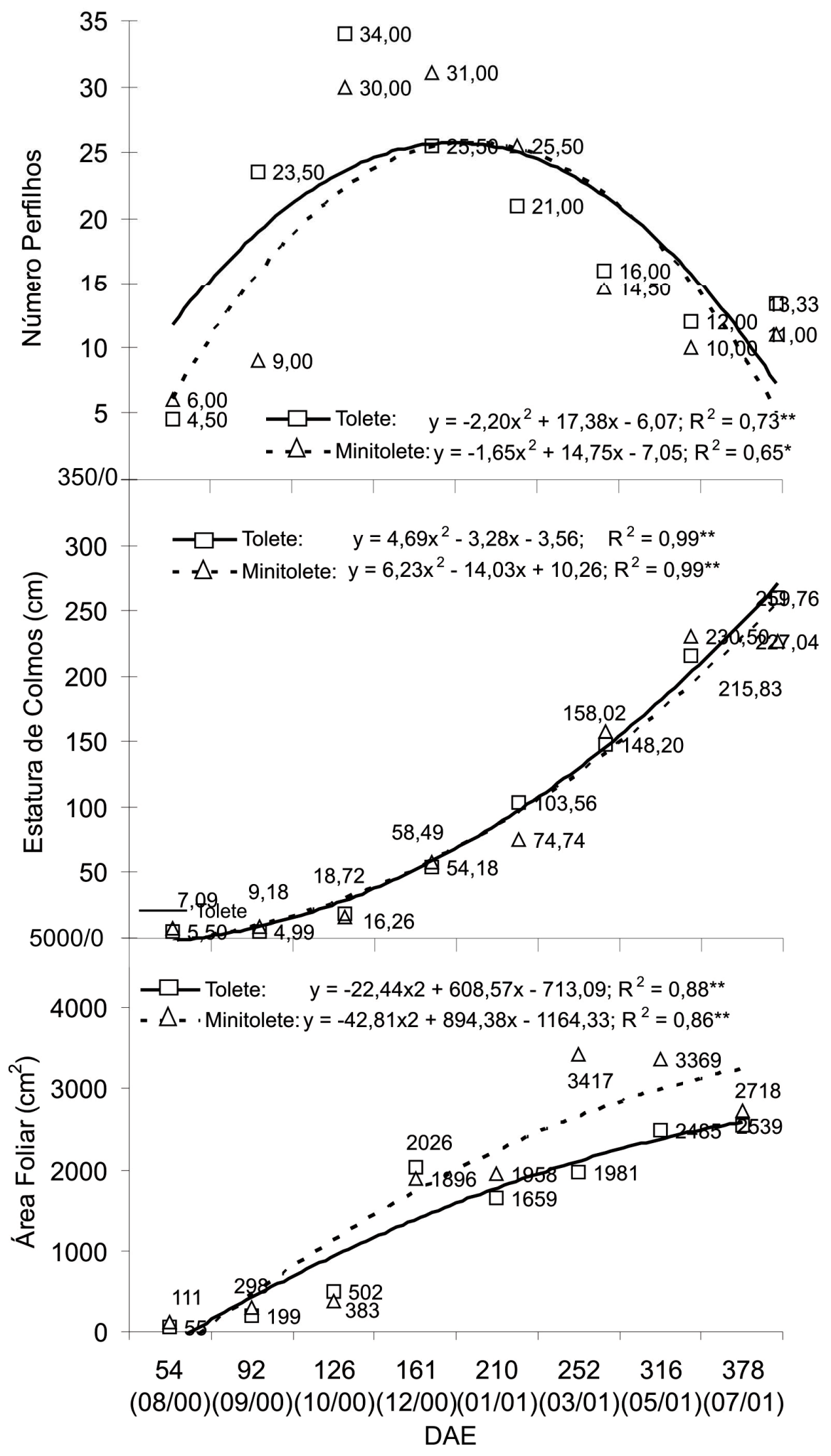

FIGURA 2 - Número de perfilhos, Estatura de colmos $(\mathrm{cm})$ e Área foliar $\left(\mathrm{cm}^{2}\right)$ em dois sistemas de plantio: minitolete e convencional, para a variedade RB855536, em oito épocas de amostragens. Estação Experimental de Paranavaí, SCA-UFPR, PR, 2000/2001. 
BUSO, P.H.M. et al. O sistema radicular da variedade...

A variedade RB855536 apresentou o máximo perfilhamento aos 126 DAC (31 de outubro) com 34,0 perfilhos $\mathrm{m}^{-2}$, para o sistema de plantio em tolete, enquanto que para o sistema de plantio em minitolete o máximo perfilhamento foi observado aos 161 DAC (dezembro) com 31,0 perfilhos $\mathrm{m}^{-2}$ (Figura 2). Ido et al. (2006), trabalhando em Rizotron com esta variedade e em sistema de plantio em minitoletes, encontraram número máximo de perfilhos (33) em outubro, sendo considerados valores elevados, comprovando a característica varietal de excelente brotação de soqueira. Neste trabalho, os autores identificaram que no mês de março ocorreu estabilização do número de perfilhos (13 perfilhos), tendo pouca mudança até o período de colheita, com queda de $61 \%$ no número de perfilhos de outubro a julho.

Neste trabalho, verificou-se que a partir dos 161 DAC para o sistema de plantio em tolete e 210 DAC para o sistema de plantio em minitolete houve morte gradativa dos perfilhos até o 378 DAC. A mortalidade entre a época de número máximo de perfilhos e a última é de $61 \%$ para o plantio em tolete e $65 \%$ para o plantio de minitoletes, em concordância com Ido et al. (2006).

$\mathrm{Na}$ Figura 2 observa-se que ambos os sistemas de plantio apresentaram comportamento semelhante quanto à emissão de perfilhos. $O$ plantio em tolete apresentou maior número de perfilhos até 161 DAC, quando as linhas de tendência se unem até 378 DAC. A equação de regressão quadrática apresentou $R^{2}$ de 0,73 para o plantio em tolete e 0,81 para o plantio em minitoletes, demonstrando boa correlação entre os dados.

Uma das características principais nesta variedade refere-se ao elevado perfilhamento. Entretanto, verificou-se que após o período de intenso perfilhamento, houve grande redução no número de perfilhos nos dois sistemas de plantio. Oliveira et al. (2007) correlacionaram o perfilhamento com a produtividade, relatando que 0 intenso perfilhamento na RB855536 indicou correlação negativa com a produtividade $\left(R^{2}=-\right.$ $\left.0,65^{*}\right)$, quando comparada com outras variedades.

Para a característica estatura de colmos, observou-se aumento lento do início da fase de crescimento até os 161 DAC. A partir desta fase e até os 210 DAC, ocorreu um período de intenso crescimento em estatura, com acréscimo de $93 \%$ e $29 \%$ para os sistemas de plantio em tolete e minitolete, respectivamente. Enquanto que na época seguinte (252 DAC, no sistema de plantio em tolete, houve crescimento de $42 \%$ e para o sistema de plantio em minitolete o crescimento foi de $111 \%$. Resultados semelhantes foram descritos por ldo et al. (2006) que relataram valores de $52 \mathrm{~cm}, 124 \mathrm{~cm}$ e $196 \mathrm{~cm}$ aos 161,210 e 252 DAC respectivamente, representando ganho de $144 \mathrm{~cm}$ nas três épocas. Oliveira et al. (2005), trabalhando com cana-deaçúcar em ciclo de ano e meio, indicaram crescimento em estatura de colmos de $179 \mathrm{~cm}$, entre os meses de dezembro e março.

Comparando-se os dois sistemas de plantio (tolete e minitolete), observou-se que a tendência dos tratamentos foi bastante semelhante, havendo pequena superioridade em estatura no final do ciclo no sistema de plantio em tolete (Figura 2).

A área foliar (AF) da variedade RB855536 foi $502 \mathrm{~cm}^{2}$ e $383 \mathrm{~cm}^{2}$ aos 126 DAC (Figura 2), passando para $2026 \mathrm{~cm}^{2}(403 \%)$ e $1896 \mathrm{~cm}^{2}$ (495\%) aos 161 DAC, nos sistemas de plantio em tolete e em minitolete, respectivamente. Ambas as épocas são as únicas em que a AF no sistema de plantio em minitolete foi inferior ao sistema de plantio em tolete. Nos meses seguintes, houve aumento na AF chegando ao máximo de $2539 \mathrm{~cm}^{2}$ no sistema de plantio em tolete aos 378 DAC e $3417 \mathrm{~cm}^{2}$ no sistema em plantio em minitolete aos 252 DAC, período que Oliveira et al. (2005) também relataram intenso aumento da AF entre os meses de janeiro a março. Ido et al. (2006) descreveram que o crescimento da parte aérea ocorre principalmente nestes meses devido às adequadas condições de temperatura e precipitação.

A superioridade na quantidade de raízes (comprimento) com o plantio de tolete aos 54 DAC (profundidade de 00-20) pode ser explicada pela grande emissão de perfilhos até 92 DAC. No plantio em minitolete, houve a formação de nove perfilhos enquanto que no plantio com tolete formaram-se 23 perfilhos. Em contrapartida, no plantio em minitolete, houve $50 \%$ mais área foliar que no plantio com tolete.

No sistema de plantio em tolete, verificouse que a estatura do colmo e o sistema radicular aumentaram, porém com perda da AF por morte dos perfilhos (210 DAC), ocasionado pelo perfilhamento excessivo e auto-sombreamento no início do desenvolvimento (Terauchi et al., 1999; Terauchi \& Matsuoka, 2000). No sistema de plantio em minitolete, neste período, houve menor perfilhamento $\mathrm{o}$ que pode ter proporcionado aumento na AF e no comprimento de raízes.

Nesse sentido Ramesh \& Mahadevaswany (2000) constataram que os cultivares que perfilham menos, além de apresentarem menores porcentagens de mortalidade, apresentaram perfilhos com maior estatura, conforme pode ser constatado para esta variedade no sistema de plantio em minitolete.

Nos dois sistemas de plantio foi identificado diminuição na quantidade de raízes aos 252 DAC. No plantio em tolete houve queda de $24 \%$ no número de perfilhos, de $44 \%$ no comprimento de raízes e aumento de $43 \%$ em estatura e de $19 \%$ em $\mathrm{AF}$, enquanto que no plantio em minitoletes houve queda de $43 \%$ no número de perfilhos, de $14 \%$ no comprimento de raízes, aumento de $111 \%$ em estatura e de $75 \%$ em AF.

\section{CONCLUSÕES}

No sistema de plantio em tolete, apesar de apresentar menor AF, houver maior concentração no crescimento das raízes em profundidade no final do ciclo, podendo ser considerada característica 
BUSO, P.H.M. et al. O sistema radicular da variedade...

importante para o desenvolvimento em condições de restrição hídrica, onde o maior crescimento radicular permitirá maior tolerância a estes períodos.

\section{AGRADECIMENTOS}

Ao Programa de Melhoramento Genético da Cana-de-açúcar/RIDESA/UFPR.

\section{REFERÊNCIAS}

1. AGUIAR, S. F. Observações sobre sistema radicular da cana-planta (Saccharum spp). 1978, 24 p. Trabalho de graduação (Graduação em Agronomia) - Faculdade de Ciências Agrárias e Veterinárias, Universidade Estadual Paulista Júlio de Mesquita Filho. Jaboticabal, 1978.

2. ASSOCIAÇÃO DE PRODUÇÃO DE BIONERGIA DO ESTADO DO PARANÁ (ALCOPAR). Estatísticas: histórico de produção da cana-de-açúcar no Brasil e no Paraná. 2009. Disponível em: <http://www.alcopar.org.br/estatisticas/ hist_prod_pr.php >. Acesso em: abril 2009.

3. $B O ̈ \bar{H} M, W$. Methods of studying root systems. Berlin: Springler, 1979. $188 \mathrm{p}$.

4. COMPANHIA NACIONAL DE ABASTECIMENTO (CONAB). Central de informações agropecuárias: safras - cana. 2009. Disponível em: < http://www.conab.gov.br/conabweb/download/safra/3_levantamento2008_dez2008.pdf >. Acesso em: abril 2009

5. HERMANN, E. R.; CÂMARA, G. M. S. Um método simples para estimar a área foliar de cana-de-açúcar. STAB. v. 17, n. 5, p. 32-34, 1999.

6. IDO, O. T. et al. Crescimento e distribuição radicial de três variedades cultivares de cana-de-açúcar, em cana-soca, em dois tipos de solo, em Rizotron, i. uso do Winrhizo. Scientia Agraria. v. 7, n. 1-2, p. 21-26, 2006.

7. OLIVEIRA, R. A. et al. Área foliar em três cultivares de cana-de-açúcar e sua correlação com a produção de biomassa. Pesquisa Agropecuária Tropical. v. 37, n. 2, p. 71-76, 2007.

8. OLIVEIRA, R. A. et al. Crescimento e desenvolvimento de três cultivares de cana-de-açúcar, em cana-planta, no Estado do Paraná. Scientia Agraria. v. 5, n. 1-2, p. 87-94, 2004.

9. OLIVEIRA, R. A. et al. Crescimento e desenvolvimento de três cultivares de cana-de-açúcar, em cana-planta, no Estado do Paraná: taxas de crescimento. Scientia Agraria. v. 6, n. 1-2, p. 85-89, 2005

10. RAMESH, P.; MAHADEVASWAMY, M. Effect of formative phase drought on different classes of shoots, shoot mortality, cane attributes, yield and quality of four sugarcane cultivars. Journal Agronomy \& Crop Science. v. 185, n. 4, p. 249258, 2000.

11. TAYLOR, D.; ARKIN, G. F. Root zone modification fundamentals and alternatives. In: TAYLOR, H. M.; ARKIN, G. F. (Ed.). Modifying the root environment to reduce crop stress. St. Joseph : ASAE, p.3-16, 1981.

12. TERAUCHI, T.; MATSUOKA, M. Ideal characteristics for the early growth of sugarcane. Japanese Journal of Crop Science. v. 69 , n. 3, p. 286-292, 2000.

13. TERAUCHI, T.et al. Comparison of the early growth between sugarcane and sweet sorghum. Japanese Journal of Crop Science. v. 68, n. 3, p. 414-418, 1999.

14. VASCONCELOS, A. C. M., CASAGRANDE, A. A., PERECIN, D. Avaliação do sistema radicular da cana-de-açúcar por diferentes métodos. Revista Brasileira de Ciência.do Solo. v. 27, n. 5, p. 849-858, 2003.

15. VASCONCELOS, A. C. M. Comportamentos de clones IAC e variedades de cana-de-açúcar (Saccharum spp.) nas condições edafoclimáticas da região do Vale do Paranapanema. 1998. 108 p. Dissertação (Mestrado em AgronomiaProdução Vegetal) - Faculdade de Ciências Agrárias e Veterinárias, Universidade Estadual Paulista Júlio de Mesquita Filho. Jaboticabal, 1998.

16. VASCONCELOS, A. C. M.; GARCIA, J. C. Cana-de-açúcar: Ambientes de Produção. Desenvolvimento Radicular da cana-de-açúcar. Ribeirão Preto: IAC/Apta, 2005, 5 p. (Encarte Técnico, 110).

Recebido em 24/07/2006 Aceito em 28/05/2009 
